\title{
Development of new basal insulin peglispro (LY2605541) ends in a disappointing result
}

\author{
Takahisa Hirose ${ }^{1}$
}

Received: 8 January 2016/Published online: 29 January 2016

(C) The Japan Diabetes Society 2016

On 4 December 2015, Eli Lilly and Company (Indianapolis, IN, USA) announced that they made the decision to end the basal insulin peglispro (BIL) development program, which was on its way to phase III trials. Stable and prolonged pharmacokinetic (PK) and pharmacodynamic (PD) profiles are very important for basal insulin, and several analog insulins for basal supplement have been developed in this decade [1]. More efficient and safer glycemic control has been realized with subsequent new basal insulins. Established long-acting basal insulins available in Japan include insulin glargine $100 \mathrm{U} / \mathrm{ml}$ [2], insulin detemir [3], insulin degludec [4], and insulin glargine $300 \mathrm{U} / \mathrm{ml}[5,6]$.

LY2605541 is a novel PEGylated basal insulin known as BIL. Insulin lispro is a $5.8-\mathrm{kDa}$ peptide hormone; 20-kDa polyethylene glycol (PEG) is a neutral linear conjugated to insulin lispro to give rise to the basal analog LY2605541. It is able to bind three molecules of water, allowing it to become highly hydrated, thereby increasing the hydrodynamic size of the molecule, which delays absorption and reduces renal filtration, resulting in a protracted half-life of LY2605541. The PEGylation process also protects against proteolytic degradation. PEGylation is novel in the context of insulin but is a well-established strategy to improve therapeutic properties of proteins, as shown for interferon. In the phase I study, LY2605541 showed flat PK and PD profiles accompanied by glucose normalization, prandial insulin

Takahisa Hirose

takahisa.hirose@med.toho-u.ac.jp

1 Division of Metabolism and Endocrinology, Department of Medicine, Toho University School of Medicine, 6-11-1 Omori-Nishi Ota-ku, Tokyo 143-8541, Japan dose reduction, and no severe hypoglycemia [7]. In addition to the long and flat PK/PD profile, LY2605541 demonstrates preferential hepatic versus peripheral action relative to insulin glargine in healthy individuals [8]. In this euglycemic clamps study, insulin glargine resulted in increased glucose disposal rate (GDR) at insulin concentrations where endogenous glucose production (EGP) was significantly suppressed. In contrast, at comparable EGP suppression, LY2605541 had minimal effect on GDR at lower doses and substantially less effect on GDR than insulin glargine at higher doses. This hepatospecific characteristic of LY2605541 may mimic physiological endogenous insulin, which is directly secreted into the portal system.

The IMAGINE phase III studies investigated LY2605541 in several patient populations [9-15]. Over all in those studies, LY2605541 led to a significantly greater reduction in $\mathrm{HbA}_{1 \mathrm{c}}$ compared with insulin glargine $100 \mathrm{U} /$ $\mathrm{ml}$, although other recently launched basal insulins reduced $\mathrm{HbA}_{1 \mathrm{c}}$ no less effectively in the treat-to-target studies [16]. In the IMAGINE 1 and 3 studies, there was a greater rate of total hypoglycemia, but lower nocturnal hypoglycemia, compared with insulin glargine $100 \mathrm{U} / \mathrm{ml}$. The increased rate of daytime hypoglycemia may be due to bolus insulin overdose after covering with strong basal insulin LY2605541. All these studies reported elevated alanine transaminases, and some studies also reported increased fat content in the liver [9-13]. Although Nippon Eli Lilly accomplished phase 3 studies in Japan, Eli Lilly and Company announced in February 2015 that regulatory submission for approval by the US Food and Drug Administration (FDA) had been delayed so that additional clinical data could be gathered to further understand and characterize the potential effects on changes in liver fat induced by LY2605541 [16]. 
Finally, on the date stated in the beginning of this report, the company announced that they made the decision to end the BIL development program, which was on its way to phase III trials. The company explained that the reason for this decision was that the investment of time and money needed to assess potential development plans addressing liver data observations in IMAGINE trials. Although liver data may not show LY2605541 to be hepatotoxic, the authors speculate that it induces liver steatosis and the probability that it induces nonalcoholic steatohepatitis ((NASH)-like status cannot be excluded. In conclusion, whereas the company's decision is disappointing, it is discerning, as it may take much time to attain liver-safety approval of LY2605541. However, the author believes that the massive amounts of data regarding liver-specific basal insulin is useful and contributes greatly to the development of future insulin therapy.

\section{Compliance with ethical standards}

Conflict of interest statement Total clinical research grant: Sanofi, Eli Lilly, Novo Nordisk, Kissei, Boehringer Ingelheim, Ono, AstraZeneca. Honoraria for lectures: Sanofi, Eli Lilly, Novo Nordisk, Merck (MSD), Dainippon-Sumitomo, Novartis, Kissei, Boehringer Ingelheim, Ono, Kissei, AstraZeneca.

Ethics policy All procedures followed were in accordance with the ethical standards of the responsible committee on human experimentation (institutional and national) and with the Declaration of Helsinki of 1964 and later versions. Informed consent or substitute for it was obtained from all patients for being included in the study.

\section{References}

1. Pettus J, Cavaiola TS, Tambrlane WV, Edelman S. The past, present, and future of basal insulins. Diabetes Metabol Res Rev. 2015. doi:10.1002/dmrr.2763 Published online.

2. Heinemann L, Linkeschova R, Rave K, Hompesch B, Sedlak M, Heise T. Time action profile of the long-acting insulin analog insulin glargine (HOE901) in comparison with those of NPH insulin and placebo. Diabetes Care. 2000;23:644-9.

3. Heise T, Pieber TR. Towards peakless, reproducible and longacting insulins. An assessment of the basal analogues based on isoglycemic clamp studies. Diabetes Obes Metab. 2007;9:648-59.

4. Kalra S. Insulin degludec: a significant advancement in ultralongacting basal insulin. Diabetes Ther. 2013;4:167-73.

5. Shiramoto M, Eto T, Irie S, et al. Single-dose new insulin glargine $300 \mathrm{U} / \mathrm{ml}$ provides prolonged, stable glycaemic control in Japanese and European people with typ 1 diabetes. Diabetes Obes Metab. 2015;17:254-60.
6. Becker RH, Nowotny I, Teichert L, Bergmann K, Kapitza C. Low within- and between-day variability in exposure to new insulin glargine 300 U/ml. Diabetes Obes Metab. 2015;17:261-7.

7. Sinha VP, Howey DC, Choi SL, Mace KF, Heise T. Steady-state pharmacokinetics and glucodynamics of the novel, long-acting basal insulin LY2605541 dosed once-daily in patients with type 2 diabetes mellitus. Diabetes Obes Metab. 2014;16:344-50.

8. Henry RR, Mudaliar S, Ciaraldi TP, Armstrong DA, Burke P, Pettus J, Garhyan P, Choi SL, Jacober SJ, Knadler MP, Quin Lam EC, Prince MJ, Bose N, Porksen N, Sinha VP, Linnebjerg H. Basal insulin peglispro demonstrates preferential hepatic versus peripheral action relative to insulin glargine in healthy subjects. Diabetes Care. 2014;37:2609-25.

9. Garg SK, Jinnouchi H, Dreyer M, et al. Greater HbA1c reduction with basal insulin peglispro (BIL) vs. insulin glargine (GL) in an open-label, randomized study in T1D patients (pts): IMAGINE 1 [abstract]. Diabetes 2015; 64(Suppl 1): A25 Abstract 95-OR.

10. Davies MJ, Russell-Jones D, Selam J-L, et al. Basal insulin peglispro (BIL) is superior to insulin glargine (GL) in reducing HbA1c at 52 wks in insulin-naïve T2D patients (pts) treated with oral antihyperglycemic medications (OAMs): IMAGINE 2 [abstract]. Diabetes 2015; 64(Suppl 1): A24 Abstract 93-OR.

11. Bergenstal RM, Lunt H, Franek E, et al. Superior reduction of HbA1c in a double-blind, randomized study of basal insulin peglispro (BIL) vs. insulin glargine (GL) in patients (pts) with T1D: IMAGINE 3 [abstract]. Diabetes 2015; 64(Suppl 1): A250 Abstract 986-P.

12. Blevins T, Pieber TR, Vega GC, Zhang S, Bastyr EJ III, Chang AM. Superior $\mathrm{HbA1c}$ reduction with basal insulin peglispro (BIL) vs. insulin glargine (GL) and preprandial insulin lispro in a double-blind study in patients (pts) with type 2 diabetes (T2D): IMAGINE 4 [abstract]. Diabetes 2015; 64(Suppl 1): A250 Abstract 985-P.

13. Buse JB, Rodbard HW, Serrano CT, et al. Superior HbA1c reduction with basal insulin peglispro (BIL) vs. insulin glargine (GL) alone or with oral antihyperglycemic medications (OAMs) in T2D patients (pts) previously treated with basal insulin: IMAGINE 5 [abstract]. Diabetes 2015; 64(Suppl 1): A249-A250 Abstract 984-P.

14. Grunberger G, Chen L, Rodríguez A, Tinahones FJ, Jacober SJ, Bue-Valleskey J. Basal insulin peglispro (BIL) provides clinically and significantly better HbAlc control with less nocturnal hypoglycemia (hypo) than NPH when used in combination with oral agents in insulin-naïve T2D patients (pts): IMAGINE 6 [abstract]. Diabetes 2015; 64(Suppl 1): A256 Abstract 1004-P.

15. Garg SK, Selam J-L, Bhargava A, et al. Similar HbA1c reduction and hypoglycemia with variable time (VT) and fixed time (FT) dosing of basal insulin peglispro (BIL) in type 1 diabetes (T1D): IMAGINE 7 [abstract]. Diabetes 2015; 64(Suppl 1): A260 Abstract 1018-P.

16. Bergenstal RM, Rosenstock J, Bastyr EJ 3rd, et al. Lower glucose variability and hypoglycemia measured by continuous glucose monitoring with novel long-acting insulin LY2605541 versus insulin glargine. Diabetes Care. 2014;37:659-65. 